\title{
Demonstrating the Elliptical Orbit of Mars using Naked Eye Data
}

\author{
Kevin Krisciunas ${ }^{1}$
}

\begin{abstract}
Over the course of 3.1 years we determined the position of Mars on 75 dates using a handheld cross staff and two to five bright reference stars of known right ascension and declination on each occasion. On average the observed positions of Mars are within \pm 11 arc minutes of the true ecliptic latitudes and ecliptic longitudes. After deriving the two dates of opposition to the Sun, we were able to carry out a two stage analytical experiment on Mars' orbit. From the 2015/2016 data we obtain a value of the eccentricity of $0.093 \pm 0.012$. The 2015/2016 data can be fit reasonably well by adopting a circular orbit for the Earth, but the 2017/2018 data must be fit with an ellipse for Mars and an ellipse for the Earth. Applying the two ellipse model to the 2015/2016 data, we obtain an RMS error of the ecliptic longitudes of only \pm 7.5 arc minutes. While Kepler was able to derive the shape of Mars' orbit while relying on data of Tycho Brahe accurate to \pm 2 arc minutes, today we may assume an ellptical orbit, and we can show that much less accurate data are consistent with an ellipse having an eccentricity equal to the modern accepted value of 0.0934 , within the errors.
\end{abstract}

Subject headings: Popular Physics, Dynamics - planetary

\section{Introduction}

The fundamental paradigm of solar system astronomy prior to the time of Copernicus was that the Earth was at the center of the solar system. Also, celestial bodies were assumed to move along perfect circles. This led to the system of deferents and epicycles. One prime motivation for the use of epicycles was to account for retrograde motion. Copernicus' great book On the Revolutions of the Heavenly Spheres (1543) asserted that the Sun is physically and truly at the center of the solar system, and that the motions of the planets, including the Earth, in a heliocentric system provide a much simpler explanation for the retrograde

\footnotetext{
${ }^{1}$ George P. and Cynthia Woods Mitchell Institute for Fundamental Physics \& Astronomy, Texas A. \& M. University, Department of Physics \& Astronomy, 4242 TAMU, College Station, TX 77843; krisciunas@physics.tamu.edu
} 
motion of the planets. However, Copernicus retained circular motion. Also, he retained the notion of epicycles to account for variations of distance of the planets from the Sun (Gingerich 1993).

In the Almagest Ptolemy gives values for the minimum and maximum angular sizes of the Moon of $31^{\prime} 20^{\prime \prime}$ and $35^{\prime} 20^{\prime \prime}$, respectively (Toomer 1984). Naked eye observations by this author have demonstrated that one can show, without using a telescope, that the angular size of the Moon varies in a regular way, implying that the Moon's distance varies in a regular way (Krisciunas 2010, 2016). The implied eccentricity of the Moon's orbit was found to be $\approx 0.04$. The true eccentricity of the Moon's orbit is 0.055 , but its orbit is anything but a simple ellipse, owing to the combined gravitational forces of the Sun and Earth.

Since the time of Hipparchus (ca. 150 BC) it has been known that the minimum Earth-Sun distance occurs each year shortly after the winter solstice.2 Using very simple observations, Lahaye (2012) derived a value of the Earth's orbital eccentricity of $0.017 \pm$ 0.001, which compares extremely well with the official modern value of 0.0167 . This was accomplished by determining the variation of the equation of time (difference of apparent solar time and mean solar time) over the course of the year using observations of the length of the shadow of a gnomon. It was also necessary to know the obliquity of the ecliptic, which is directly obtained from such observations on the first day of summer and the first day of winter (Krisciunas et al. 2012). The point here is that it was certainly known in ancient times and at the time of Copernicus that orbiting bodies are not equidistant from the bodies they orbit.

In 1609 Johannes Kepler published the original versions of his first two laws of planetary motion: 1) the orbit of a planet is an ellipse, with the Sun at one focus; and 2) what we now call the law of areas, that the radius vector of a planet sweeps out equal areas in equal times. The Second Law can be stated as follows:

$$
r^{2} \mathrm{~d} \theta=\mathrm{h}
$$

where $r$ is the distance between a planet and the Sun, $\mathrm{d} \theta$ is an angular increment in radians,

\footnotetext{
${ }^{2}$ Hipparchus determined that the maximum Earth-Sun distance, the "solar apogee" in a geocentric model, occurs when the Sun's ecliptic longitude is 5.5 degrees east of the boundary between Taurus and Gemini (Toomer 1981, on p. 211). This means the ecliptic longitude of the Sun at the time of the mininum EarthSun distance is roughly $90+5.5+180=275.5 \mathrm{deg}$. Accounting for the observed advance of the perihelion of Earth's orbit of $11.45 \mathrm{arc}$ "/yr (Fitzpatrick 2011), one finds that the Earth's perihelion occurs nowadays in the first few days of January. For a table of the Earth's perihelion and aphelion from 2001 to 2100 see: http://www.astropixels.com/ephemeris/perap2001.html.
} 
and $h$ is a constant unique to each planet.

Newton's breakthroughs in mathematics and mechanics led to the realization that Kepler's First Law needed correction. The very center of the Sun is not at the focus of a planetary orbit. A planet orbits the center of mass of the planet-Sun system, and the Sun orbits that center of mass too (Carroll \& Ostlie 2007, chapter 2). This idea, of course, has led to the discovery of many extra-solar planets via the radial velocity method.

In the autumn of 2015 Mars was nicely situated in the constellation Leo before sunrise. We began a sequence of observations of Mars using a simple cross staff (Fig. 1) ? $^{3}$

Say the full width of the cross staff is $d$, and suppose at a linear distance $D$ down the ruler the angular separation of two celestial objects exactly matches the width of the cross staff. Then the angular separation of the two objects will be

$$
\theta=2 \tan ^{-1}\left(\frac{\mathrm{d}}{2 \mathrm{D}}\right)
$$

If an observer can measure the angular separation of a planet and two stars of known celestial coordinates, there are two possible solutions for the position of the planet, one on each side of the great circle arc joining the two stars. If the planet is close to being on the great circle arc between the two stars, perhaps no solution results, given errors of measurement. If the positions of a planet and the two stars form a spherical triangle with reasonably equal sides, this is ideal, and the planetary position can be determined reasonably accurately. We found that if only two reference stars are used, and the observations are not carefully made, the systematic errors of the two angular distances can conspire to give a planetary position that is in error by more than one degree. We found it advisable to use three to five reference stars. We assume a system of accurate stellar coordinates of bright stars along the zodiac. We adopt the J2000.0 coordinates of such stars from the SIMBAD database 4

In Fig. 2 we show a nearly ideal set of reference stars distributed around the location of Mars on 21 November 2018 UT. In our experience the most accurate angular separations are obtained when the reference stars are brighter than fourth magnitude and the angular

${ }^{3} \mathrm{~A}$ pattern for making the cross staff can be obtained from this link: https://sites.google.com/a/uw.edu/introductory-astronomy-clearinghouse/labs-exercises/measuringangular-sizes-and-distances. The reader should note that when printed out the scale may look like inches, but is, in fact, somewhat different.

${ }^{4}$ http://simbad.u-strasbg.fr/simbad/ 
separation, as measured with our cross staff, is between 9 and 15 degrees.

Understanding Johannes Kepler's efforts to discover the elliptical nature of Mars' orbit requires serious effort. A good place to start is an article by Gingerich (1989). Kepler endeavored to determine the true shape of a planetary orbit. Is it an offset circle, a combination of a larger circle and one epicycle (as fit by Copernicus), an ovoid, or an ellipse? To make a long story shorter, using data obtained by Tycho Brahe and his assistants, Kepler found systematic differences amounting to $8^{\prime}$ between the measured ecliptic longitudes of Mars and his favored model (an ovoid). These $8^{\prime}$ differences occurred when Mars was located in the octants of its orbit (45 degrees either side of the Sun, or 135 degrees either side). Since Tycho's data were demonstably accurate to $\pm 2^{\prime}$ or better, Kepler decided that there was a problem with the model. This led him to conclude that the true orbital shape was an ellipse.

We wondered if it is possible to demonstrate from simple naked eye observations that the orbit of Mars is indeed an ellipse. Or, requiring less rigor, are the positions of Mars consistent with an elliptical orbit? If so, can we derive the eccentricity? Here we present results based on two seasons of observing spanning 410 days and 391 days, respectively.

A full blown orbital determination for the planet Mars is beyond the scope of the present paper. That would involve simultaneously solving for all six orbital elements. We only endeavor to show that a dataset obtained with simple equipment can be fit with an ellipse of eccentricity $\approx 0.093$. Other values of the eccentricity can be shown to give ecliptic longitudes that differ from the observational data by a few degrees, far larger systematic differences than the internal random errors of the observations.

\section{Data Acquisition}

In Table 1 we give various data relating to Mars. For each Julian Date we give the "true" right ascension $(\alpha)$ and declination $(\delta)$ of the planet, obtained using an algorithm of van Flandern \& Pulkkinen (1979). These coordinates are accurate to $\pm 1^{\prime}$. Note that these coordinates will correspond to the equinox of date. To convert these coordinates to ecliptic latitude $(\beta)$ and longitude $(\lambda)$ we need the following formulas from spherical trigonometry (Smart 1977, p. 40):

$$
\begin{aligned}
& \sin (\beta)=\sin (\delta) \cos (\epsilon)-\cos (\delta) \sin (\alpha) \sin (\epsilon) \\
& \sin (\lambda)=\frac{\cos (\delta) \sin (\alpha) \cos (\epsilon)+\sin (\delta) \sin (\epsilon)}{\cos (\beta)}
\end{aligned}
$$




$$
\cos (\lambda)=\frac{\cos (\delta) \cos (\alpha)}{\cos (\beta)} .
$$

$\epsilon$ is the obliquity of the ecliptic, $23^{\circ} 26^{\prime} 21^{\prime \prime} .406$ for the year 2000. Using the ATAN2 function in FORTRAN or Python with arguments $\sin (\lambda)$ and $\cos (\lambda)$, we obtain the ecliptic longitude in the correct quadrant.

Table 1 also gives the observed right ascension, declination, ecliptic longitude, and ecliptic latitude of Mars, derived from the cross staff measurements, along with the number of reference stars used and the value for each date of the Sun's ecliptic longitude. The values of the Sun's longitude were calculated using the second method of Meeus (1988, p. 80). On occasion we desired one more sufficiently bright reference star and used the derived position of Saturn or Jupiter as the extra reference "star".

Consider two celestial objects with equatorial coordinates $\left(\alpha_{1}, \delta_{1}\right)$ and $\left(\alpha_{2}, \delta_{2}\right)$. The angular separation $(\theta)$ between two objects is:

$$
\cos (\theta)=\sin \left(\delta_{1}\right) \sin \left(\delta_{2}\right)+\cos \left(\delta_{1}\right) \cos \left(\delta_{2}\right) \cos \left(\alpha_{1}-\alpha_{2}\right) .
$$

Next consider a spherical quadrilateral that is bounded by starting and ending right ascensions, and starting and ending declinations. The quadrilateral is divided into a grid, given a nominal increment in each coordinate of 0.01 deg. We used a computer program of our devising that uses the coordinates of two reference stars and the measured angular distance of a planet from each of these stars to determine the coordinates of the planet. This is done by brute force, determining which positions in the quadrilateral match the angular distances to the two stars, within some settable error. If the coordinates of the stars are J2000.0 coordinates, then the derived right ascension and declination of the planet are also J2000.0 coordinates.

The derived ecliptic coordinates of Mars for 2015/2016 are shown in Figure 3. The solid line in the plot shows the locus of "true" positions from van Flandern \& Pulkkinen (1979). The most obvious thing to note is that there is a variation of the ecliptic latitude of Mars. This means that its orbit is inclined to the orbit of the Earth. At different oppostions, Mars shows different retrograde patterns on the sky. In this paper we will only be analyzing the ecliptic longitudes of Mars vs. time.

Given that most positions of Mars listed in Table 1 were derived from angular separations with respect to three to five reference stars, almost all out nightly mean derived right ascensions and declinations have easy-to-calculate internal random errors. These are 
sometimes as small as $\pm 0.01 \mathrm{deg}$ (which we do not really believe). On one occasion (JD $2457399.9840)$ the internal random error for right ascension was $\pm 0.23 \mathrm{deg}$ and the internal random error of declination was $\pm 0.47 \mathrm{deg}$. Typical internal random errors for right ascension and declination are $\sigma_{\alpha} \approx \sigma_{\delta} \approx \pm 0.10 \mathrm{deg}$.

The most objective measure of the accuracy of our data would be the scatter of the nightly mean positions with respect to some to-be-determined model. However, since we have "true" positions of Mars from van Flandern \& Pulkkinen (1979) we can also make a direct estimate of the accuracy of our observations. The easiest way to do this is to precess the ecliptic longitudes from column 4 in Table 1 to equinox J2000 by subtracting 50.25 arc seconds per year times the number of years from JD 2,457,543.5 (0.0 January 2000) to the date of observation. The ecliptic latitudes require no precession correction.

For the 2015/2016 season the standard deviations of the distributions of differences are: $\sigma_{\lambda}= \pm 0.119 \mathrm{deg}\left(7.1^{\prime}\right)$ for ecliptic longitude and $\sigma_{\beta}= \pm 0.178 \mathrm{deg}\left(10.7^{\prime}\right)$ for ecliptic latitude. The square root of the sum of squares of those errors is $\sigma_{t o t}= \pm 0.214 \mathrm{deg}$, or $12.8^{\prime}$, which we may consider the minimum value of the effective accuracy of our cross staff measurements. For the $2017 / 2018$ season we find $\sigma_{\lambda}= \pm 0.263 \mathrm{deg}\left(15.8^{\prime}\right)$ and $\sigma_{\beta}= \pm 0.201 \mathrm{deg}\left(12.1^{\prime}\right)$. Combining the two seasons' data we find $\sigma_{\lambda}= \pm 0.185 \mathrm{deg}\left(11.1^{\prime}\right)$ and $\sigma_{\beta}= \pm 0.188 \mathrm{deg}$ $\left(11.3^{\prime}\right)$. We are not sure why the second season's data are, at face value, less accurate than the data of the first season, but the distribution and usefulness of sufficiently bright reference stars is not the same for all zodiacal constellations.

\section{Fitting the Data}

In Book 5, Chapter 19, of On the Revolutions of the Heavenly Spheres Copernicus (1543) derives the perigee, mean, and apogee distances of Mars. He obtains the values 1.374, 1.520, and 1.649 AU, respectively. Thus, Copernicus knew the amount by which Mars' distance from the Sun varies, and his mean distance is very close to the modern value of the semimajor axis size of Mars' orbit (1.52366 AU). He used the combination of one large circle and one smaller circle, akin to a deferent and an epicycle.

Let us consider the elliptical orbit of Mars. The equation of an ellipse is:

$$
r=\frac{a(1-e)}{1+e \cos \theta}
$$

where $\mathrm{r}$ is the Mars-Sun distance, $a$ is the semi-major axis of the ellipse, $e$ is the eccentricity, and angle $\theta=0$ when Mars is at perihelion. 
The velocity along the orbit (Carroll \& Ostlie 2007, Eq. 2.36) is

$$
v^{2}=G\left(M_{\odot}+M_{\text {Mars }}\right)\left(\frac{2}{r}-\frac{1}{a}\right) .
$$

Since the mass of Mars is $\approx 3.23 \times 10^{-7} \mathrm{M}_{\odot}$ (Tholen, Tejfel, \& Cox 2000, p. 295), for our purposes here we shall ignore it.

We wish to calculate the position of Mars at increments of one Earth day starting at the moment of its perihelion. At perihelion $\mathrm{r}=\mathrm{r}_{\min }=a(1-e)$. Using the known semi-major axis size and eccentricity of Mars' orbit (or a range of assusmed values), we can calculate the maximum velocity at perihelion with Equation 8. On perihelion day traveling at velocity $v_{\max }$ Mars moves 0.6349 degrees along its orbit as viewed from the Sun. This allows us to calculate the constant $h$ for Mars using Equation 1. Then, by alternating use of Equations 7 and 1 we can calculate $r$ and $\theta$ for Mars each day along its orbit. The X-Y coordinates are obtained simply: $X=r \cos (\theta)$ and $Y=r \sin (\theta)$.

Given the small eccentricity of the Earth's orbit (0.0167), let us begin with the simplest possible model by assuming a circular orbit for the Earth, which of course implies a constant velocity. On average, the Earth moves 360.0/365.2422 $=0.985647$ degrees per day with respect to the Sun. This gives us another set of X-Y coordinates in the same coordinate system, with the Sun at the origin and the $+\mathrm{X}$-axis in the direction of Mars' perihelion.

Consider Fig. 4. According to the Solar Systems Dynamic Group, Horizons On-Line Ephemeris System at Jet Propulsion Laboratory 5 one perihelion of Mars occurred on Julian Date 2,457,003.8524 (12 December 2014, at 08:27:29 UT). The next perihelion occurred on Julian Date 2,457,691.0507 (29 October 2016, at 13:13:04 UT). For the moment let us take the perihelion dates as given. They are not directly observable, but the date of opposition of Mars essentially is. Mars' ecliptic longitude differs from that of the Sun by 180 deg near the mid-time of its retrograde motion. From our data we find opposition to have occurred at JD 2,457,730.36 \pm 0.62 (21 May 2016 at 21 hours UT). According to the Astronomical Almanac for the Year 2016, opposition occurred on May 22nd at 11 hours UT, or JD 2,457,530.96. The agreement is within one standard deviation.

Mars' 2016 opposition occurred 526.9 days after the perihelion of 12 December 2014. Call it 527 days. The X-Y coordinates of the day-by-day position of Mars in our coordinate system give $\theta=265.545 \mathrm{deg}$ on the day of opposition. Since the Earth moves $0.985647 \mathrm{deg}$ per day along its orbit, the 269th pair of Earth coordinates gives an angle $\theta$ most closely

${ }^{5}$ https://ssd.jpl.nasa.gov/horizons.cgi 
matching that of Mars (265.139 vs. 265.545 deg, in fact). Given the index $i$ of the Earth's coordinates, the corresponding index of Mars' coordinates for the same day is equal to $i+$ $(527-269)$.

We wrote a simple Python program that calculates the X-Y coordinates of Mars for each day starting at perihelion, and the X-Y coordinates of the Earth. With the appropriate offset of the indices of the two sets of coordinates we can obtain the direction toward Mars from the Earth for any given date. This generates a locus of "some angle" vs. time in days. We then used a FORTRAN program originally written for fitting templates to supernova light curve photometry to adjust that locus to the dates and ecliptic longitudes of our Mars observations. This produces a goodness of fit parameter equal to the sum of squares of differences between the template and the data (in other words, like $\chi^{2}$ minimization, but with equal weights for all the points). The square root of the goodness of fit parameter divided by the number of data points minus two gives the RMS residual.

We tried values of the eccentricity of Mars' orbit ranging from 0.053 to 0.133. As shown in the bottom panel of Fig. 5 , for $\mathrm{e}=0.053$ the observed residuals are $+1.0 \mathrm{deg}$ at the start, $-1.3 \mathrm{deg}$ at JD 2,457,624, and $+3.4 \mathrm{deg}$ at the end. For $\mathrm{e}=0.133$ the observed residuals are -1.9 deg at the start, but +1.9 deg at JD 2,457,600, and -1.3 deg at the end. A fit with $\mathrm{e}=0.0934$ (the modern accepted value) is much better. The 2015/2016 dataset and the use of a circular orbit for the Earth give a most likely value of eccentricity of $0.0930 \pm$ 0.012. Thus, our simple model allows us to show that data much less accurate than Tycho's are consistent with an elliptical orbit for Mars, and one having an eccentricity equal to the modern accepted value, within the errors.

For the best fit the RMS residual is $\pm 0.297 \mathrm{deg}\left(17.8^{\prime}\right)$. There is a trend to the residuals. The are primarily negative at the start, positive at the end, with the final residual equal to +1.0 deg - a $3-\sigma$ outlier. What improvements can result from a model that adopts an elliptical shape for the Earth's orbit, with e $=0.0167$ ? To do this we need to know the day of the year when opposition occurs (which we have already determined) and the day of the year of the Earth's perihelion, which was 3 January 2016 to the nearest day. We then rotate the grid of X-Y coordinates of the position of the Earth so that the angle from the Sun towards the Earth matches the angle of the Sun towards Mars on opposition day, since all three must line up on that day. The differences $X_{\text {Mars }}-X_{\text {Earth }}$ and $Y_{\text {Mars }}-Y_{\text {Earth }}$ are passed to the ATAN2 function as the two arguments. We generate a locus lasting more than 410 days, as that is the extent of our dataset. The resultant locus is then shifted using the template fitting program to give the best fit of the model to the data. Using the modern accepted values of the eccentricity of Mars, time of perihelion in 2014, and semi-major axis size for Mars, this gives an RMS residual of $\pm 7.5^{\prime}$. See Fig. 6 . 
In Fig. 7 we show the observed positions of Mars from the 2017/2018 season, along with the values derived from the algorithm of van Flandern \& Pulkkinen (1979). Clearly, the retrograde loop is quite different than that of the 2015/2016 season (Fig. 3). We note that in 2015/2016 the observed duration of retrograde motion of Mars was 73.34 days. Mars backed up $16.41 \pm 0.26 \mathrm{deg}$. In 2017/2018 retrograde motion lasted only 61.92 days, and Mars backed up $10.65 \pm 0.26 \mathrm{deg}$. Knowing this, we could predict that a locus that fits the ecliptic longitudes of 2015/2016 cannot just be shifted in time and angle to fit the ecliptic longitudes of $2017 / 2018$.

For the 2017/2018 season the opposition of Mars was observed to occur at JD 2,458,325.14 \pm 0.96 (25.64 July $2018 \mathrm{UT}$ ). According to the Astronomical Almanac for the Year 2018, opposition occurred on 27.21 July 2018 UT, or JD 2,458,326.71. The agreement is within 1.64- $\sigma$.

Fig. 8 shows two fits for the 2017/2018 data. The simpler model, using a uniform circular orbit for the Earth, clearly does not work. The residuals vary from $-9.0 \mathrm{deg}$ at the start to $+7.7 \mathrm{deg}$ after opposition. The RMS residual is $\pm 6.2 \mathrm{deg}$. For our second season of data we must use elliptical orbits for both Mars and the Earth. This fit (the solid line in Fig. 8) gives an RMS residual for the ecliptic longitudes of $\pm 0.202 \mathrm{deg}\left(12.1^{\prime}\right)$.

Why does the simpler model not work for the 2017/2018 data? Consider Fig. 4. In 2018 the aphelion of the Earth occurred on July 6th. Opposition of Mars occurred on July 25 (observed) or July 27 (true), just 19 to 21 days later. So the Earth was moving almost as slowly as it does along its orbit. Mars was at perihelion on 16 September 2018 at 07:54 UT ${ }^{6}$ At opposition it was $33 \mathrm{deg}$ short of being at perihelion, meaning that Mars was moving just about as fast along its orbit as possible. During 2015/2016 Mars was near the minor axis of its orbit at the time of opposition, moving at a velocity close to its mean velocity along its orbit. A circular orbit for the Earth gave a reasonably good fit, but the trend of the residuals over a timespan greater than one year motivated us to improve the fitting by also using an elliptical orbit for the Earth.

\section{Conclusions}

Our value of the orbital eccentricity of Mars $(0.093 \pm 0.012)$, derived using the simpler fit (a circular Earth orbit), compares well with the accepted modern value (0.0934). While our data are not accurate enough to prove that Mars' orbit is an ellipse, if we fit the data

\footnotetext{
${ }^{6}$ https://in-the-sky.org/news.php?id=20180916_12_100
} 
with an ellipse, we can show that the eccentricity must be near 0.09. Thus, a dataset based on naked eye observations accurate to 0.2 or $0.3 \mathrm{deg}$ in ecliptic longitude can be shown to be in agreement with Kepler's First Law.

During 2018 the opposition of Mars occurred not long after Earth's aphelion and less than two months before Mars' perihelion. These conditions obliged us to fit the ecliptic longitudes of Mars of our second season using an elliptical orbit for Mars and an elliptical orbit for Earth. Refitting the 2015/2016 ecliptic longitudes of Mars using an ellipse for Mars' orbit and an ellipse for the Earth gives an RMS uncertainty of $\pm 7.5^{\prime}$. We regard this as a suprisingly accurate result given the primitive nature of our simple cross staff.

We made use of the SIMBAD database, operated at CDS, Strasbourg, France.

\section{REFERENCES}

Carroll, B. W., \& Ostlie, D. A. 2007, An Introduction to Modern Astrophysics, 2nd ed., San Francisco: Pearson/Addison-Wesley

Copernicus, N. 1543, On the Revolutions of the Heavenly Spheres, translated by A. M. Duncan, Newton Abbot, London, Vancouver: David \& Charles, 1976, pp. 265-266

Fitzpatrick, R. 2011, http://farside.ph.utexas.edu/teaching/336k/Newtonhtml/node115.html (accessed 26 December 2018)

Gingerich, O. 1989, in The General History of Astronomy, Vol. 2, Planetary astronomy from the Renaissance to the rise of astrophysics, Part A: Tycho Brahe to Newton, R. Taton and C. Wilson, eds., Cambridge: Cambridge Univ. Press, pp. 54-78

Gingerich, O. 1993, in The Eye of Heaven: Ptolemy, Copernicus, Kepler, New York: American Institute of Physics, pp. 193-204

Krisciunas, K. 2010, Amer. J. Phys., 78, 834

Krisciunas, K. 2016, A Guide to Wider Horizons, Dubuque, Iowa: Kendall-Hunt, pp. 41-43

Krisciunas, K., DeBenedictis, E., Steeger, J., Bischoff-Kim, A., Tabak, G., \& Pasricha, K. 2012, Amer. J. Phys., 80, 429

Lahaye, T. 2012, arXiv:1207.0982 
Meeus, J. 1988, Astronomical Formulae for Calculators, 4th ed., Richmond, Virginia: Willman-Bell

Smart, W. M. 1977, Textbook on Spherical Astronomy, 6th ed., Cambridge: Cambridge Univ Press

Tholen, D. J., Tejfel, V. G., \& Cox, A. N. 2000, in Allen's Astrophysical Quantities, 4th ed., A. N. Cox, ed., New York, Berlin, Heidelberg: Springer, pp. 293-313

Toomer, G. J. 1984, Ptolemy's Almagest, Berlin: Springer-Verlag, pp. 254, 284

Toomer, G. J. 1981, "Hipparchus," in Dictionary of Scientific Biography, Charles Coulston Gillespie, ed., New York: Charles Scribner's Sons, vol. 15, pp. 207-224

Van Flandern, T. C. \& Pulkkinen, K. F. 1979, ApJS, 41, 391 
Table 1. Mars Data

\begin{tabular}{|c|c|c|c|c|c|c|c|c|c|c|}
\hline $\mathrm{JD}^{\mathrm{a}}$ & $\mathrm{RA}_{\text {true }} \mathrm{b}^{\mathrm{b}}$ & $\mathrm{DEC}_{\text {true }}{ }^{\mathrm{b}}$ & $\lambda^{\mathrm{c}}$ & $\beta^{\mathrm{d}}$ & $\mathrm{RA}_{o b s}$ & $\mathrm{DEC}_{o b s}$ & $\lambda_{o b s}$ & $\beta_{o b s}$ & $\mathrm{~N}^{\mathrm{e}}$ & $\lambda_{\odot}$ \\
\hline 7338.9674 & 180.3206 & 1.4016 & 179.737 & 1.413 & 180.31 & 1.68 & 179.62 & 1.67 & 2 & 229.748 \\
\hline 7344.9931 & 183.6448 & -0.0205 & 183.353 & 1.430 & 183.35 & & 183.03 & 1.42 & 4 & 235.817 \\
\hline 7346.9708 & 184.7339 & -0.4856 & 184.538 & 1.435 & 184.58 & -0.26 & 184.31 & 1.59 & 2 & 237.812 \\
\hline 7359.9819 & 191.8332 & -3.48 & 192.243 & 1.467 & 191.65 & -3.57 & 192.11 & 1.32 & 4 & 250.977 \\
\hline 7364.9833 & 194.5444 & -4.6133 & 195.173 & 1.477 & 194.12 & -4.34 & 194.68 & 1.57 & 3 & 256.053 \\
\hline 7370.9813 & 197.7750 & -5.9320 & 198.651 & & 197.45 & & 198.36 & & 4 & 262.149 \\
\hline 7373.9674 & 199.3779 & -6.5758 & 200.370 & 1.492 & 199.19 & -6.50 & 200.17 & 1.49 & 4 & 265.187 \\
\hline 7380.9542 & 203.1074 & -8.0433 & 204.351 & 1.501 & 203.01 & -8.09 & 204.28 & 1.42 & 4 & 272.301 \\
\hline 7387.9889 & 206.8359 & -9.4620 & 208.301 & 1.507 & 206.51 & -9.44 & & & 4 & 279.469 \\
\hline 7391.9813 & 208.9367 & -10.2376 & 210.512 & 1.509 & 208.73 & & & 1.55 & 4 & 283.538 \\
\hline 7399. & 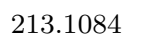 & -11.7237 & 21 & 1. & - & -11 & & & 4 & 694 \\
\hline 7406.9861 & 216.7 & -12.9 & 218. & & & -12.77 & & & 4 & 298.986 \\
\hline 7410.9840 & 218.7 & -13.6 & 220. & & & & & & - & 894 \\
\hline 7415. & 221 & -14.3 & 223. & & & 9 & & & $t_{1}$ & 990 \\
\hline 7425.9861 & 226 . & -15.8 & 228.149 & & & -15 & & 1.50 & 5 & 318.128 \\
\hline 7429.9715 & 227.9932 & -16.3574 & 230.034 & & & -16.34 & & 1.43 & 5 & 322.164 \\
\hline 7435.9937 & 230.7561 & -17.0 & 232.782 & 1.417 & 26 & -17 & & 1.34 & 5 & 328.250 \\
\hline 7443.9931 & 234.2464 & -17.9769 & 236.225 & 1.3 & 23 & -17 & & 1.28 & 5 & 336.312 \\
\hline 7449.9799 & 236 & -18.5 & & & & -18 & & 1.02 & 5 & 342.326 \\
\hline 7451.9677 & 237.4743 & -18.7441 & 239.383 & 1.305 & 236.99 & -19 & 239.01 & 0.88 & 5 & 344.318 \\
\hline 746 & 120168 & 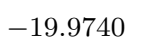 & 244.661 & & & . & & & 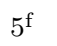 & 280 \\
\hline 747 & 244 & -20 & & & & 9 & & & $5^{f}$ & 228 \\
\hline & & -20.71 & & & & & & 0.80 & $5^{\mathrm{f}}$ & 13.132 \\
\hline 7499.9549 & 247.1279 & -21.45 & 248.792 & 0.316 & 246 & -21 & & 0.16 & 4 & 31.773 \\
\hline 7512.9493 & 245.2332 & -21.7269 & 247.097 & -0.235 & 244.72 & -21.72 & 246.63 & -0.31 & 5 & 44.407 \\
\hline & & -21 & & & & & & & 5 & 47.304 \\
\hline & & & & & & & & & $5^{\mathrm{f}}$ & 69.160 \\
\hline 7547.6240 & 233.4 & -21 & 236 & -1 & & -21 & & -2 & 3 & 77.768 \\
\hline 7555.6153 & 231.4404 & -21.0834 & 234.404 & -2.282 & & -21.20 & & -2.50 & 5 & 85.406 \\
\hline 7564.6441 & 230.1675 & -21.0421 & 233.243 & -2.541 & 229.98 & -20.87 & 233.03 & -2.42 & 5 & 94.022 \\
\hline 7569 & 229.9735 & -21.1 & $x$ & -2.6 & 7 & -21 & & -3 . & 5 & 98.770 \\
\hline 7576.6399 & 230.3070 & -21.3033 & 233.436 & -2.760 & 230.12 & -21.13 & & -2.64 & 5 & 105.460 \\
\hline 7582.6149 & 231.1228 & -21.56 & 234.238 & -2.825 & 230.94 & -21 & 234.02 & -2.68 & 5 & 111.158 \\
\hline 759 & & -22.1 & & -2.8 & & & & & 5 & 119.759 \\
\hline 7597.6517 & 235.0988 & -22.5434 & 238.054 & -2.891 & 234.89 & -22.38 & 237.83 & -2.78 & $4^{\mathrm{f}}$ & 125.512 \\
\hline 7602.6056 & 236.9372 & -22.9283 & 239.795 & -2.889 & 236.56 & -23.13 & 239.50 & -3.16 & $5^{\mathrm{f}}$ & 130.249 \\
\hline 7624.6229 & 247.6334 & -24.6631 & 249.743 & -2.783 & 247.18 & -24.45 & 249.30 & -2.64 & 5 & 151.392 \\
\hline 7633.5979 & 252.9 & -25.24 & & -2.7 & & & & & 5 & 160.063 \\
\hline 7639.5740 & 256.7638 & -25.5420 & 258.065 & -2.649 & 256.41 & -25.57 & 257.75 & -2.71 & 5 & 165.857 \\
\hline 7646.5729 & 261.4329 & -25.7851 & 262.284 & -2.575 & 261.01 & -25.79 & 261.90 & -2.60 & 5 & 172.665 \\
\hline & 266.2981 & -25 & 26 & . & 26 & -2 & & & 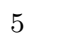 & 179.495 \\
\hline 7660.6153 & 271.3719 & -25.8399 & 271.236 & -2.407 & 270.84 & -26.06 & 270.76 & -2.62 & 5 & 186.403 \\
\hline
\end{tabular}


Table 1 -Continued

\begin{tabular}{|c|c|c|c|c|c|c|c|c|c|c|}
\hline $\mathrm{JD}^{\mathrm{a}}$ & $\mathrm{RA}_{\text {true }} \mathrm{b}$ & $\mathrm{DEC}_{\text {true }} \mathrm{b}^{\mathrm{b}}$ & $\lambda^{\mathrm{c}}$ & $\beta^{\mathrm{d}}$ & $\mathrm{RA}_{o b s}$ & $\mathrm{DEC}_{o b s}$ & $\lambda_{o b s}$ & $\beta_{o b s}$ & $\mathrm{~N}^{\mathrm{e}}$ & $\lambda_{\odot}$ \\
\hline 7665.5632 & 275.0146 & -25.7023 & 274.521 & -2.342 & 274.62 & -25.78 & 274.16 & -2.41 & 5 & 191.269 \\
\hline 7677.5806 & 275.0688 & -24.9923 & 282.737 & -2.173 & 283.87 & -24.62 & 282.59 & -1.79 & 4 & 203.147 \\
\hline 7689.5389 & 293.2365 & -23.7374 & 291.184 & -1.991 & 293.00 & -23.67 & 290.98 & -1.89 & 3 & 215.049 \\
\hline 7709.5990 & 308.6071 & -20.4297 & 305.801 & -1.660 & 308.14 & -20.52 & 305.36 & -1.64 & 3 & 235.175 \\
\hline 7714.5486 & 312.3536 & -19.3998 & 309.471 & -1.575 & 312.04 & -19.75 & 309.09 & -1.83 & 5 & 240.170 \\
\hline 7721.5361 & 317.5932 & -17.8184 & 314.683 & -1.453 & 317.24 & -18.03 & 314.39 & -1.59 & 4 & 247.239 \\
\hline 7748.5375 & 337.2173 & -10.5854 & 335.016 & -0.981 & 337.22 & -10.29 & 335.13 & -0.71 & 4 & 274.685 \\
\hline 8085.9910 & 201.8528 & -8.0165 & 203.186 & 1.070 & 201.60 & -7.87 & 202.90 & 1.11 & 3 & 246.441 \\
\hline 8127.9736 & 227.2510 & -16.7716 & 229.459 & 0.856 & 227.20 & -16.81 & 229.42 & 0.81 & 4 & 289.151 \\
\hline 8142.0066 & 236.0592 & -19.0221 & 238.137 & 0.742 & 235.67 & -19.34 & 237.85 & 0.35 & 5 & 303.441 \\
\hline 8149.9903 & 241.1237 & -20.1117 & 243.031 & 0.665 & 240.79 & -20.09 & 242.72 & 0.63 & $4^{\mathrm{g}}$ & 311.555 \\
\hline 8189.9872 & 266.6963 & -23.3068 & 266.966 & 0.098 & 266.46 & -23.08 & 266.74 & 0.32 & 4 & 351.854 \\
\hline 8197.9688 & 271.7025 & -23.4952 & 271.561 & -0.065 & 271.58 & -22.90 & 271.46 & 0.53 & 4 & 359.803 \\
\hline 8212.9583 & 280.8410 & -23.5000 & 279.933 & -0.434 & 281.06 & -23.45 & 280.14 & -0.40 & 4 & 14.635 \\
\hline 8225.9274 & 288.3395 & -23.2071 & 286.811 & -0.832 & 288.25 & -23.25 & 286.72 & -0.86 & 4 & 27.366 \\
\hline 8235.9472 & 293.7655 & -22.8568 & 291.804 & -1.198 & 293.80 & -22.65 & 291.87 & -1.00 & $3^{\mathrm{f}}$ & 37.139 \\
\hline 8257.7462 & 303.9944 & -22.0331 & 301.243 & -2.205 & 303.69 & -22.25 & 300.92 & -2.35 & 4 & 58.232 \\
\hline 8304.9201 & 312.7415 & -23.1844 & 308.798 & -5.317 & 312.24 & -23.44 & 308.28 & -5.44 & 5 & 103.360 \\
\hline 8312.9441 & 311.5969 & -23.9966 & 307.564 & -5.818 & 311.13 & -24.05 & 307.14 & -5.76 & 5 & 111.011 \\
\hline 8332.6215 & 306.5237 & -25.8726 & 302.611 & -6.474 & 305.93 & -26.20 & 302.01 & -6.67 & 4 & 129.802 \\
\hline 8337.6104 & 305.1761 & -26.1587 & 301.359 & -6.468 & 304.24 & -26.43 & 300.47 & -6.54 & 4 & 134.579 \\
\hline 8345.6142 & 303.4003 & -26.3727 & 299.749 & -6.317 & 302.88 & -26.55 & 299.25 & -6.39 & 4 & 142.257 \\
\hline 8361.5858 & 302.2008 & -25.9168 & 298.792 & -5.639 & 301.54 & -26.08 & 298.17 & -5.67 & 4 & 157.648 \\
\hline 8367.5785 & 302.6835 & -25.4859 & 299.311 & -5.311 & 302.42 & -25.52 & 299.07 & -5.29 & 4 & 163.451 \\
\hline 8387.5573 & 307.5650 & -23.2487 & 304.169 & -4.155 & 307.30 & -23.41 & 303.89 & -4.25 & 5 & 182.927 \\
\hline 8399.6042 & 312.3694 & -21.3794 & 308.954 & -3.486 & 312.25 & -20.89 & 308.98 & -2.98 & 4 & 194.776 \\
\hline 8418.5285 & 321.6447 & -17.7297 & 318.389 & -2.538 & 321.26 & -17.78 & 318.02 & -2.47 & 4 & 213.554 \\
\hline 8425.5278 & 325.4224 & -16.1759 & 322.313 & -2.223 & 325.46 & -16.25 & 322.32 & -2.31 & 4 & 220.549 \\
\hline 8443.5750 & 335.6485 & -11.7441 & 333.160 & -1.499 & 335.40 & -11.77 & 332.92 & -1.44 & 5 & 238.697 \\
\hline 8449.5701 & 339.1479 & -10.1586 & 336.938 & -1.286 & 339.30 & -9.97 & 337.15 & -1.17 & 5 & 244.757 \\
\hline 8454.5538 & 342.0836 & -8.8063 & 340.131 & -1.119 & 342.02 & -8.61 & 340.15 & -0.91 & 5 & 249.805 \\
\hline 8463.6052 & 347.4590 & -6.2888 & 346.018 & -0.839 & 345.93 & -6.28 & 345.93 & -0.79 & 4 & 258.993 \\
\hline 8476.5948 & 355.2602 & -2.5803 & 354.627 & -0.485 & 355.10 & -2.56 & 354.49 & -0.40 & 5 & 272.214 \\
\hline
\end{tabular}

Note. - Except for columns 1 and 10, all values are measured in degrees. A text file containing the data, along with similar data for Venus, Jupiter, and Saturn, can be obtained via http://people.physics.tamu.edu/public_html/planets.txt

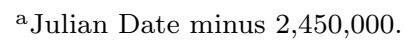

${ }^{\mathrm{b}}$ True right ascension and declination for equinox of date, using algorithm of van Flandern \& Pulkkinen (1979). Accurate to $\pm 1^{\prime}$.

${ }^{\mathrm{c}}$ True ecliptic longitude for equinox of date. To compare column 4 values to those in column 8 , the column 4 values must be precessed to equinox J2000.0 by subtracting $\approx 0.230^{\circ}(50.25$ arc seconds per year) for the $2015 / 2016$ observing season, and $\approx 0.258^{\circ}$ for the $2017 / 2018$ observing season. 
${ }^{\mathrm{d}}$ True ecliptic latitude. These can be compared directly with the values in column 9 , without any precession.

${ }^{e}$ Number of reference stars used.

f One of the reference "stars" used was Saturn, based on the position derived from our observations.

gOne of the reference "stars" used was Jupiter, based on the position derived from our observations. 
Fig. 1. - The cross staff. The cardboard cross piece slides up and down the yardstick. Using simple geometry we can use this device to determine the angular separation of two objects in the sky.

Fig. 2.- Position of Mars on 20/21 November 2018 with respect to five bright stars in Capricornus and Aquarius. The lengths of the "spokes" of the wheel are equal to the angular separations of Mars and the five stars, as measured with the cross staff. Except for $\lambda$ Aqr plus $\beta$ Aqr and $\lambda$ Aqr plus $\delta$ Cap, the other seven combinations of two reference stars can be used to determine the position of Mars.

Fig. 3.- Observed positions of Mars from 12 November 2015 to 26 December 2016 UT. The solid line shows the true positions, as derived using the algorithm of van Flandern \& Pulkkinen (1979).

Fig. 4. - The outer locus represents the orbit of Mars. The X-axis lies along the major axis of Mars' ellipse. The inner locus represents the orbit of the Earth. Mars was observed to be at opposition on 22 May 2016 and 25 July 2018. Mars' perihelion position is labeled "p".

Fig. 5.- Observed positions of Mars in 2015/2016 (upper panel). We show three fits to the data, using values of eccentricity for Mars' orbit of 0.053, 0.093, and 0.133. The Earth's orbit is assumed to be circular. The residuals of the three fits are shown in the bottom panel and indicate that the fit with eccentricity 0.093 is clearly the best of the three.

Fig. 6.- Observed positions of Mars in 2015/2016 and a fit to the data assuming elliptical orbits for Mars and the Earth. The RMS residual of the fit is $\pm 0.125 \mathrm{deg}$ ( 7.5 arc minutes).

Fig. 7.- Observed positions of Mars from 28 November 2017 to 24 December 2018 UT. The solid line shows the true positions, as derived using the algorithm of van Flandern \& Pulkkinen (1979).

Fig. 8.- Observed positions of Mars in 2017/2018 and two fits. The dashed line was calculated assuming a circular orbit for the Earth. The solid line was calculated assuming elliptical orbits for Mars and the Earth. The RMS residual of the two ellipse fit is \pm 0.202 $\operatorname{deg}(12.1$ arc minutes). 


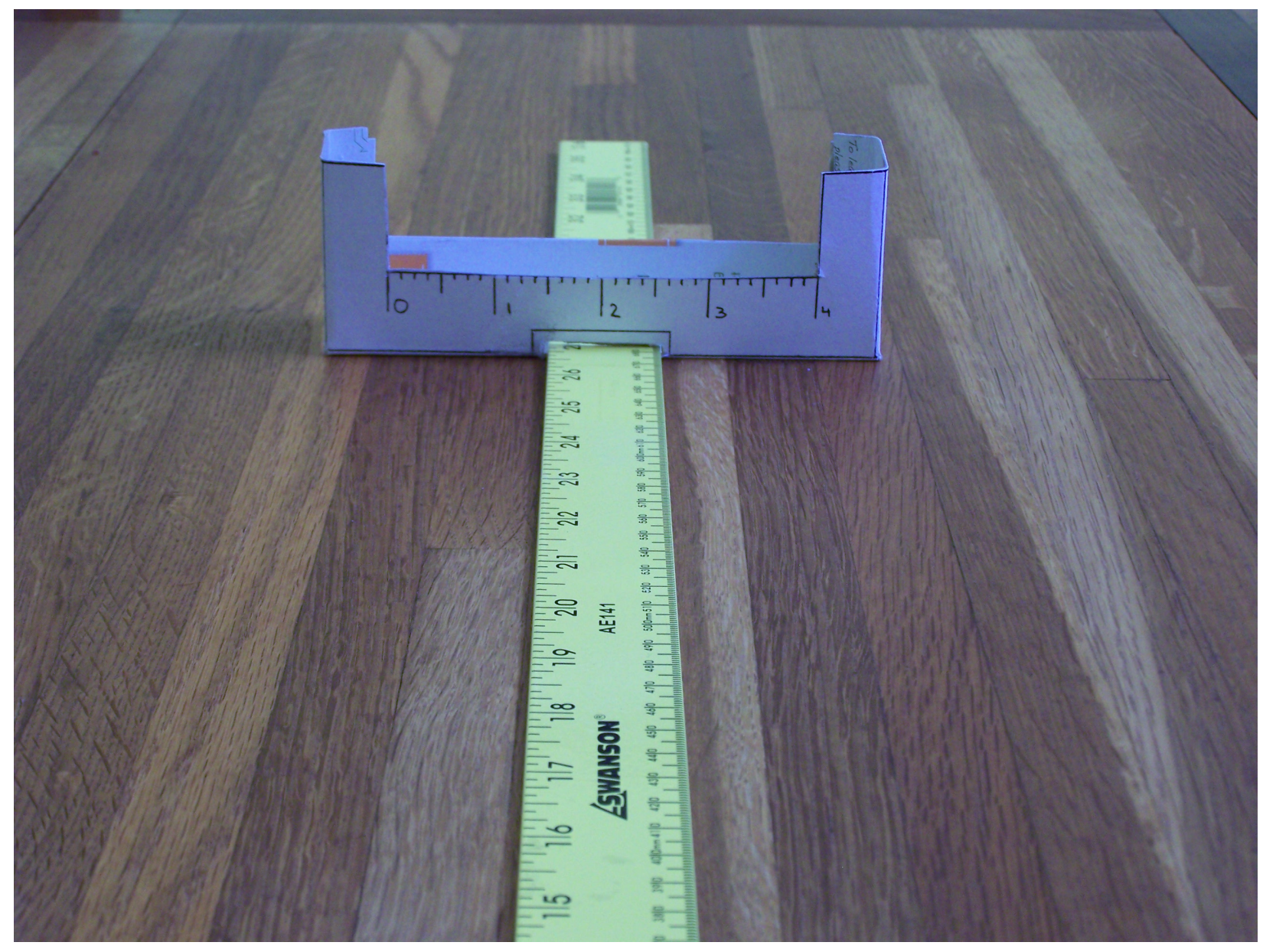

Krisciunas Fig. 1. 


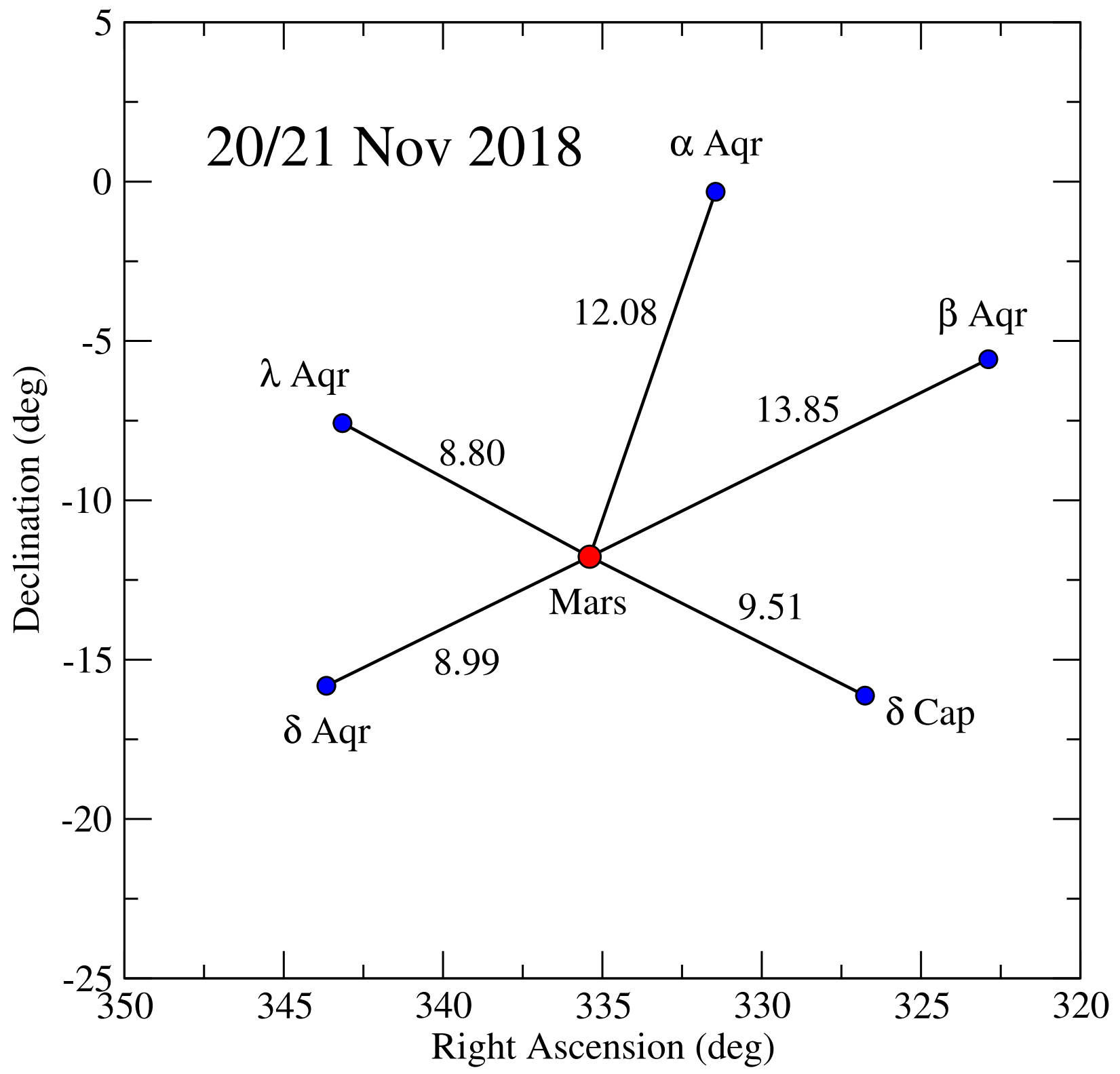

Krisciunas Fig. 2. 


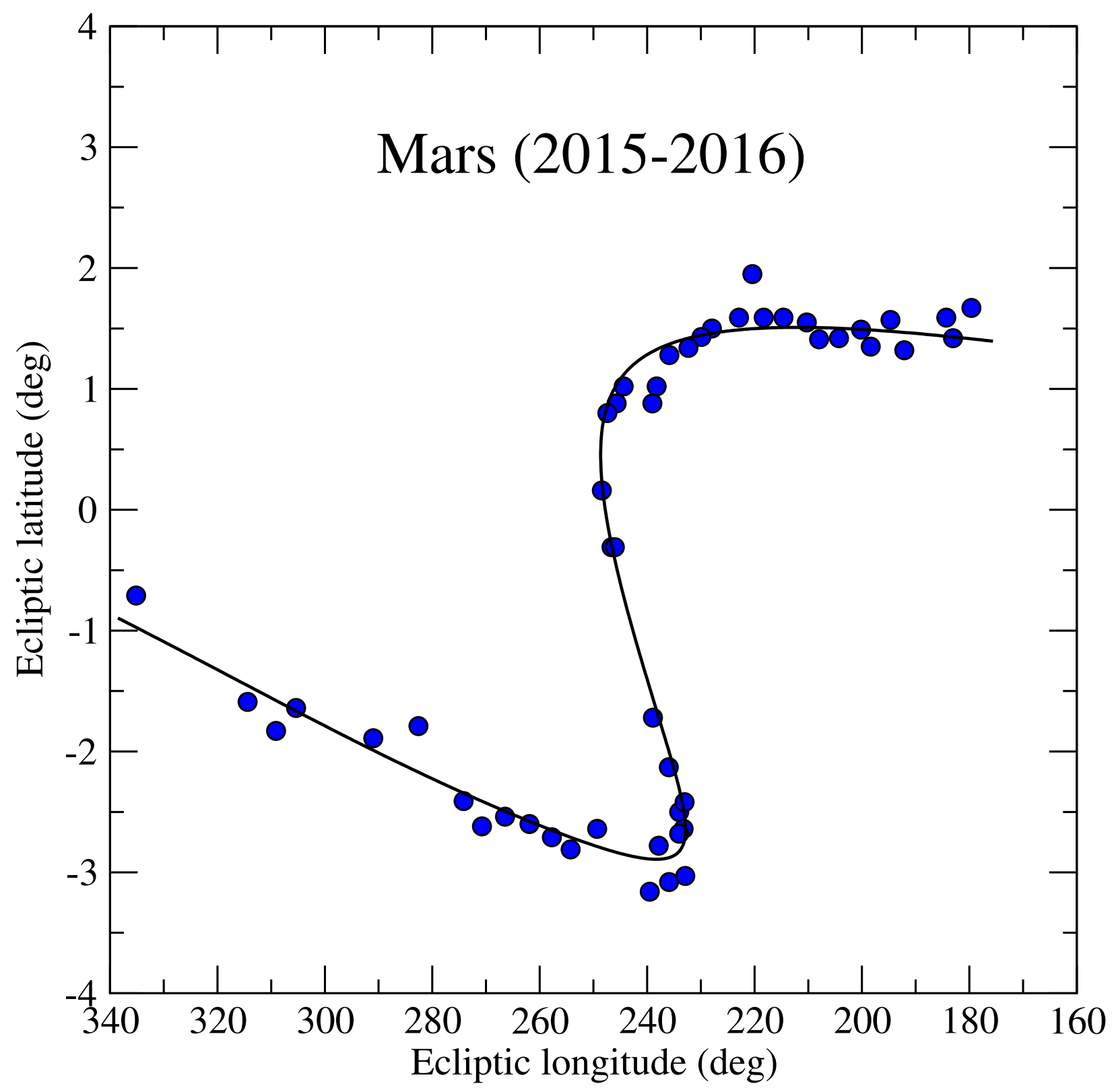

Krisciunas Fig. 3. 


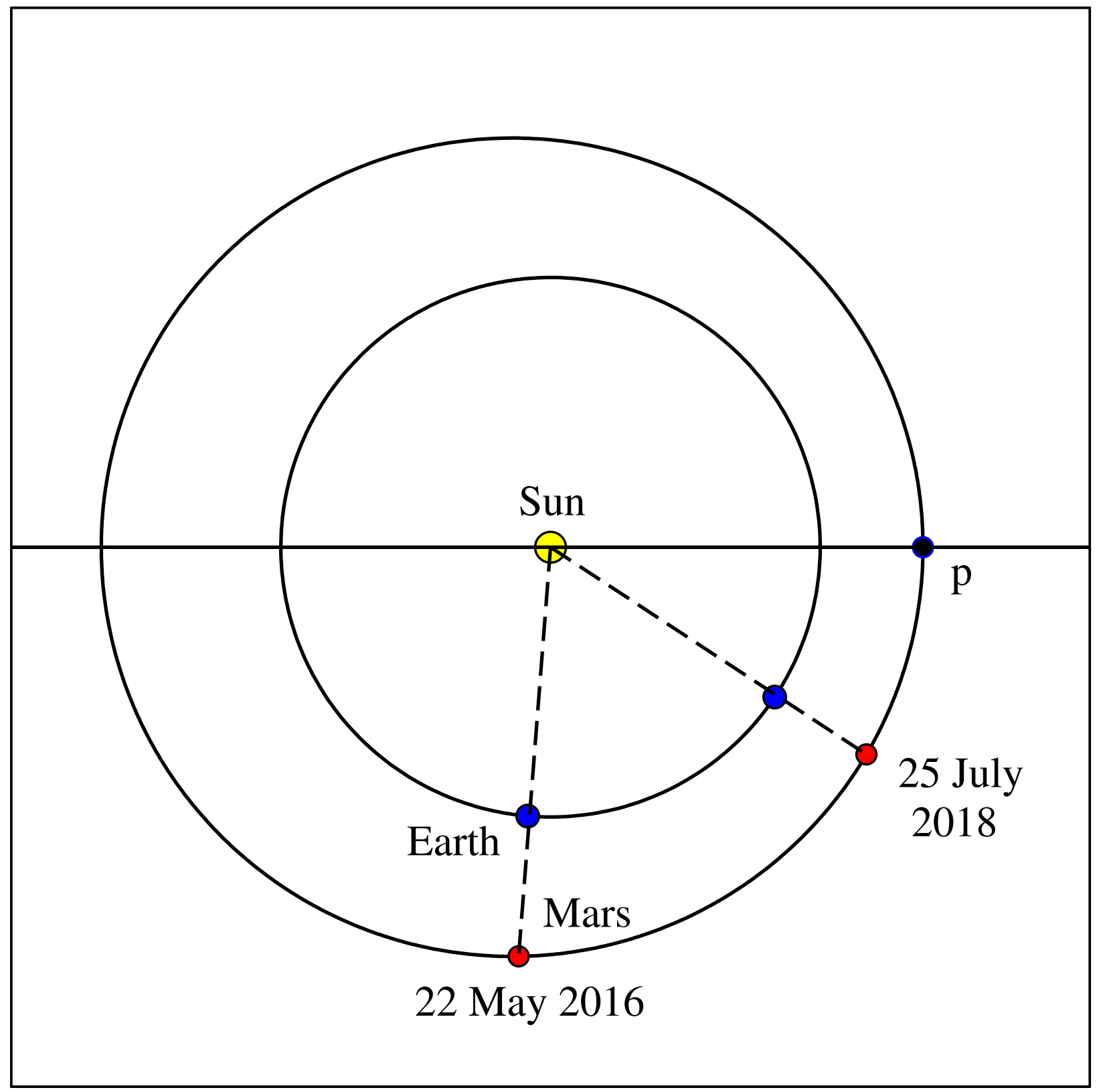

Krisciunas Fig. 4 . 


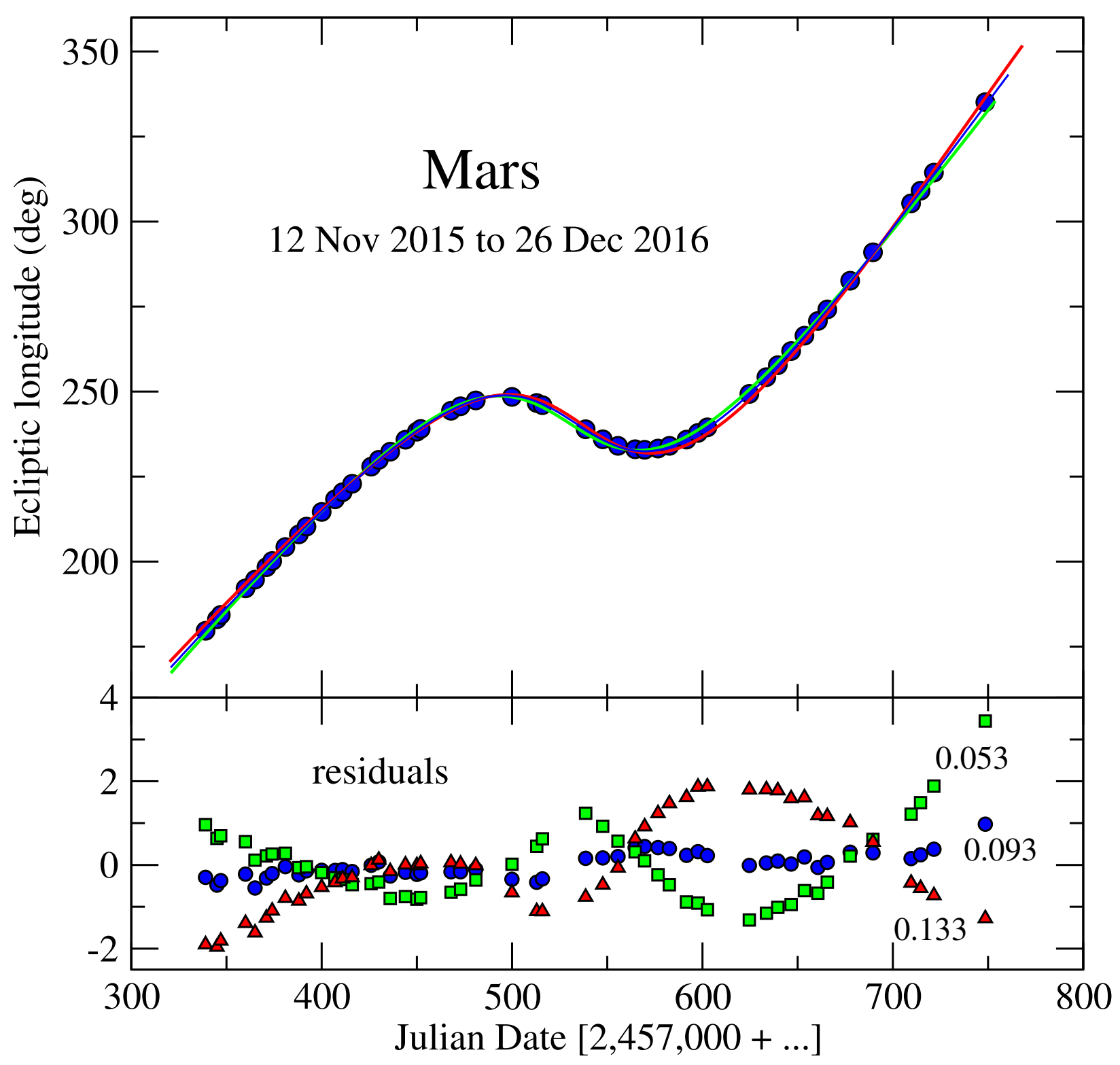

Krisciunas Fig. 5 . 


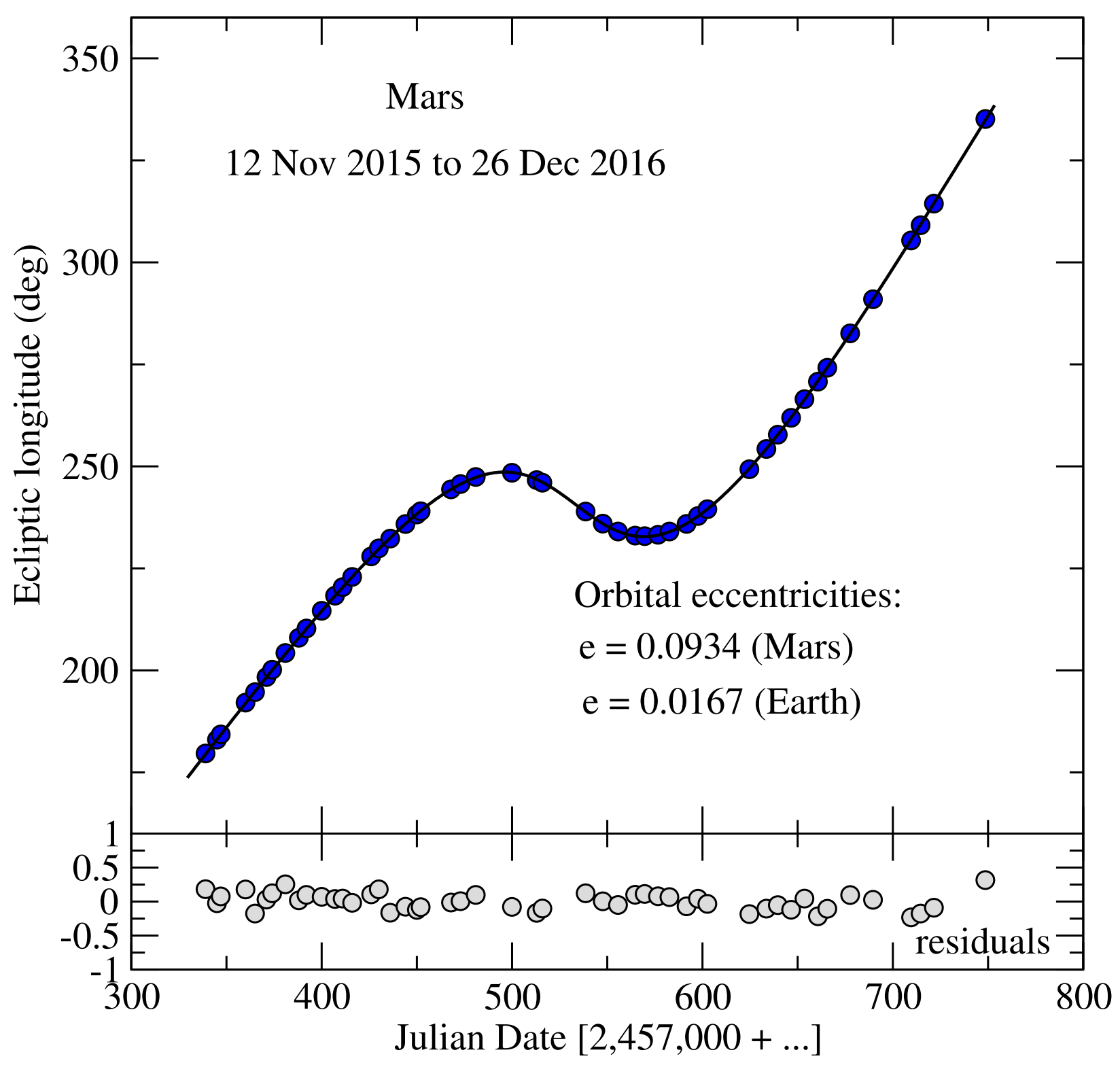

Krisciunas Fig. 6 . 


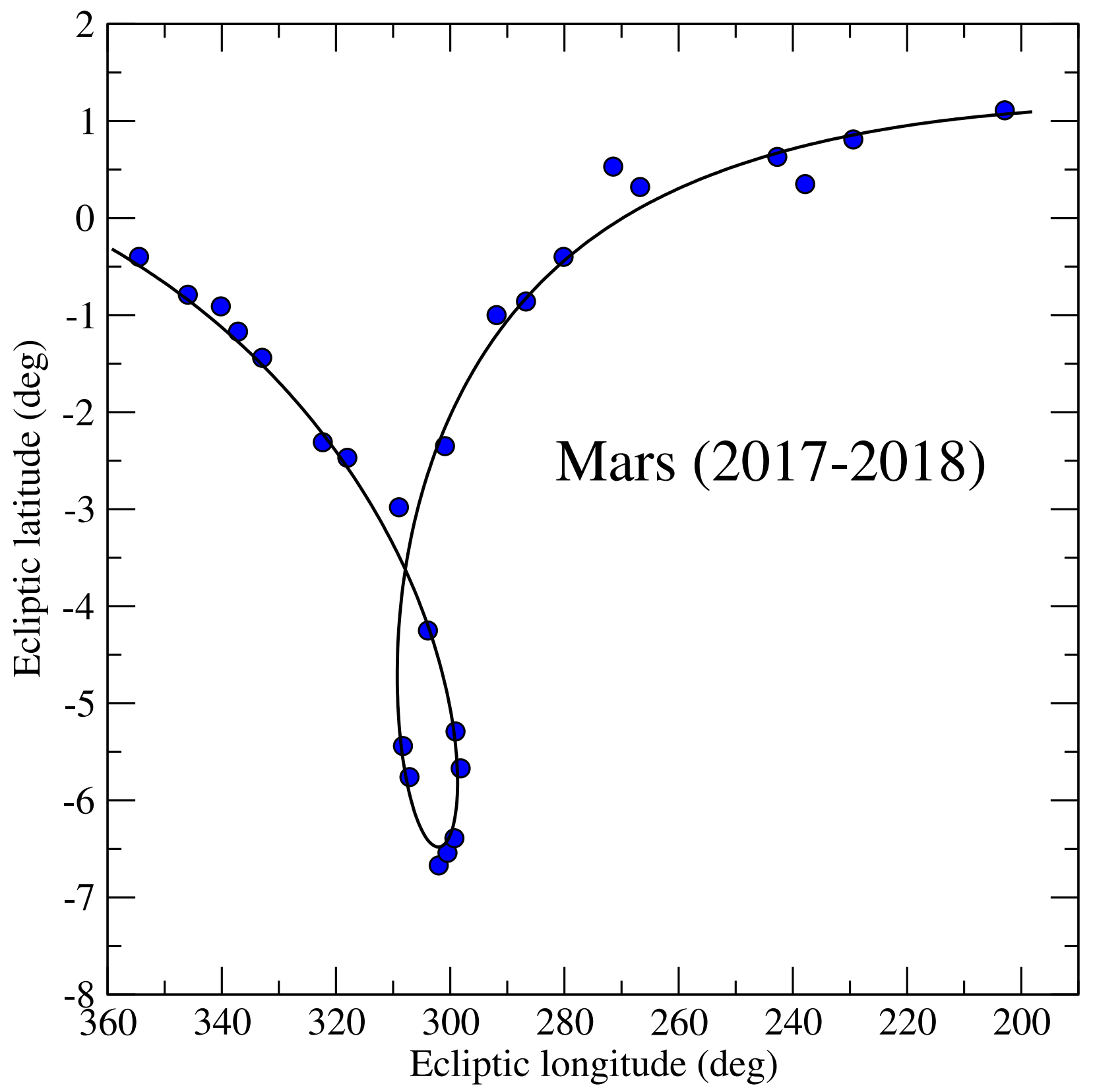

Krisciunas Fig. 7. 


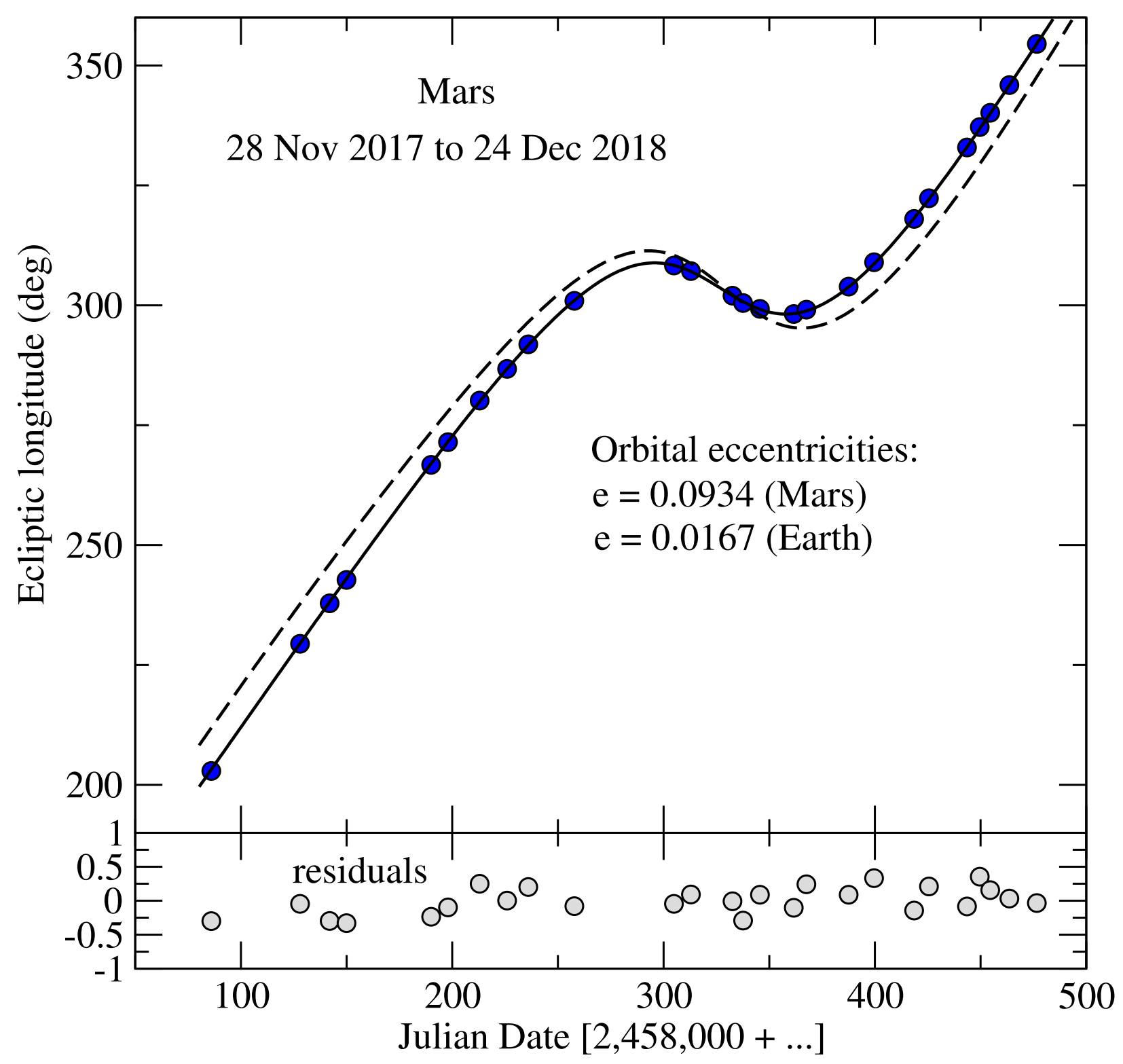

Krisciunas Fig. 8 , 